\title{
Graphene Oxide Causes Disordered Zonation Due to Differential Intralobular Localization in the Liver
}

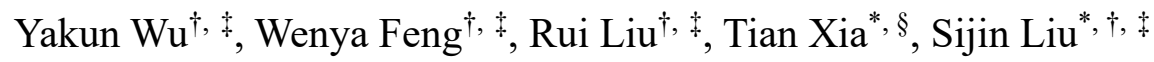

$\dagger$ State Key Laboratory of Environmental Chemistry and Ecotoxicology, Research Center for Eco-Environmental Sciences, Chinese Academy of Sciences, Beijing 100085, China

$\$$ University of Chinese Academy of Sciences, Beijing 100049, China

$\S$ Division of Nanomedicine, Department of Medicine, California NanoSystems Institute, University of California, Los Angeles, California 90095, United States.

*: Corresponding authors: Sijin Liu and Tian Xia. E-mail: sjliu@rcees.ac.cn or txia@ucla.edu. 


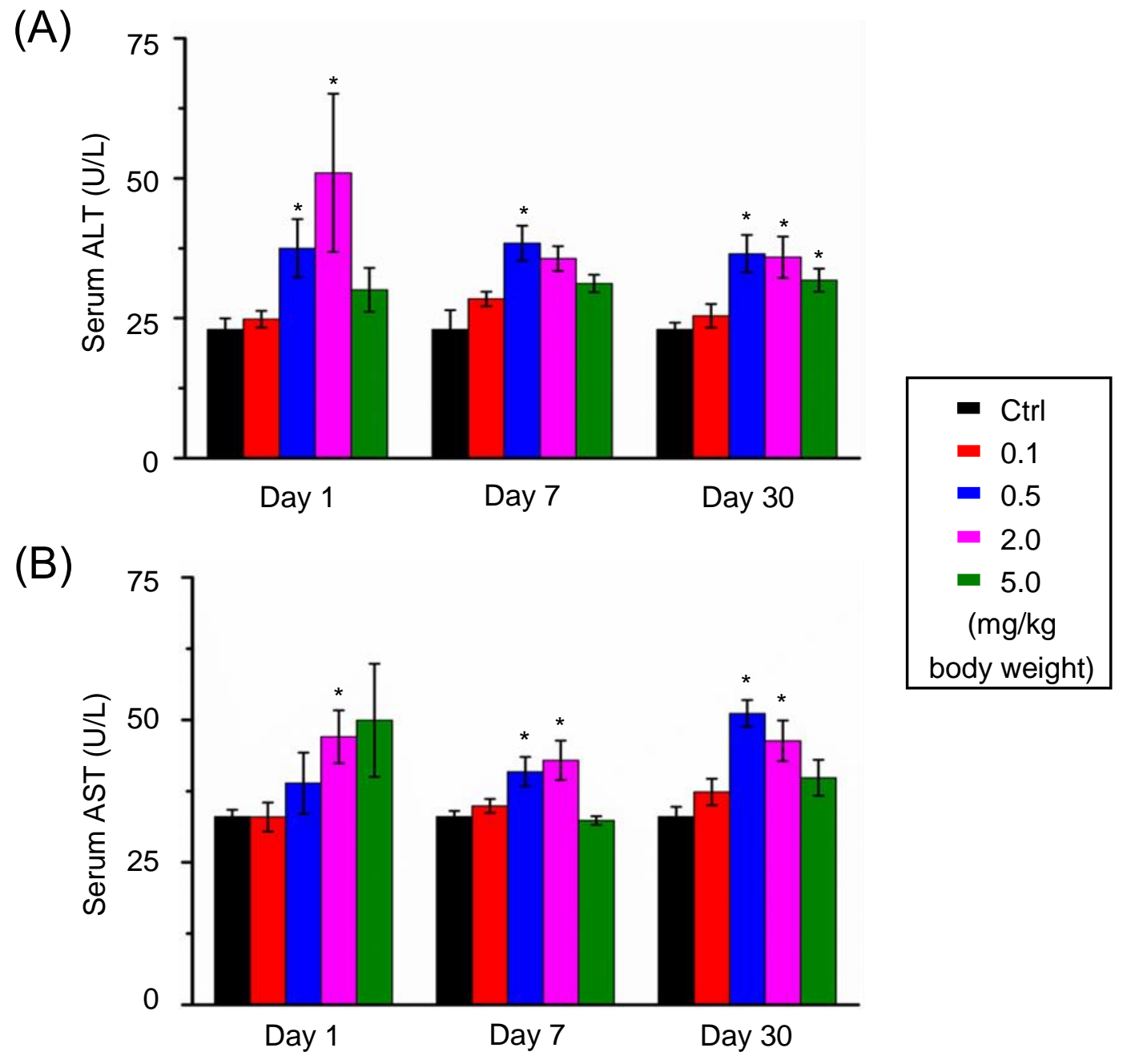

Figure S1. Serum ALT (A) and (B) AST levels in GO-exposed mice at various concentrations for indicted time $(\mathbf{n}=\mathbf{4 - 6}) . *: P<0.05$, compared to untreated control. 


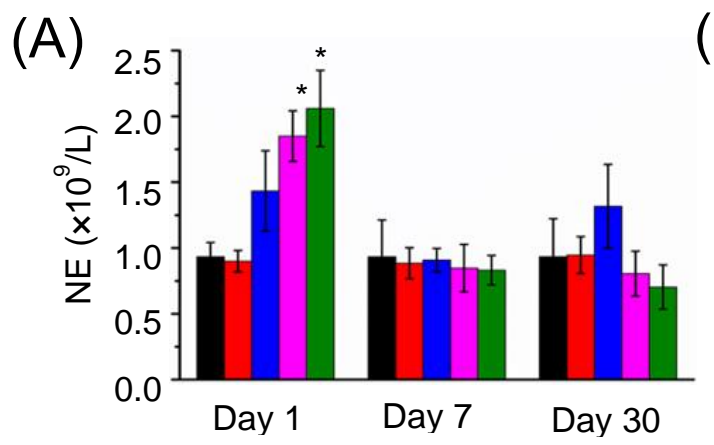

(B)

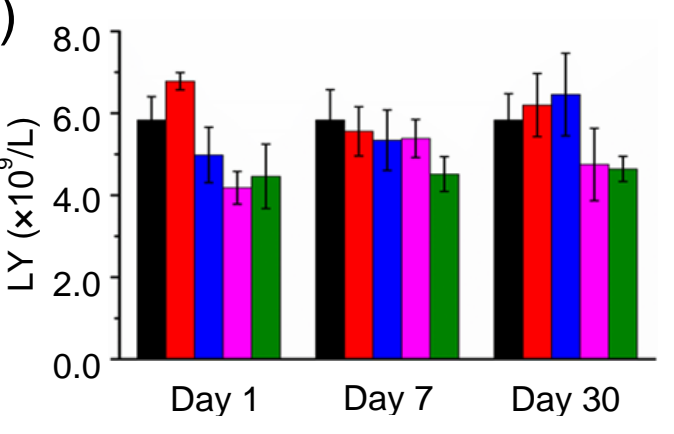

(C)

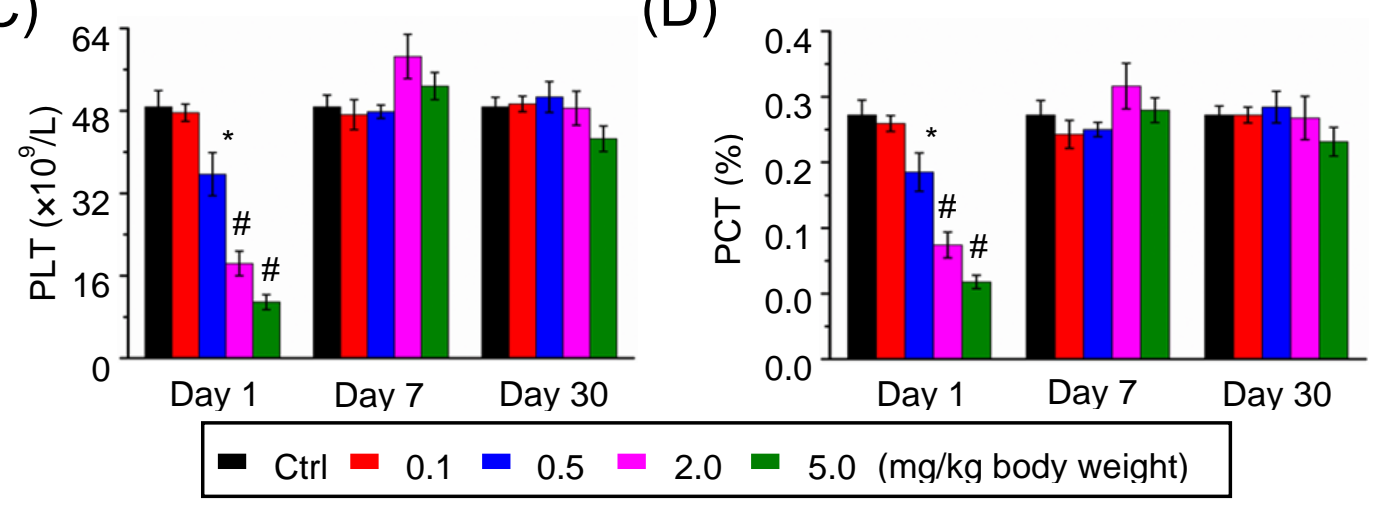

Figure S2. Hematological parameters in GO-exposed mice at various concentrations for indicted time. (A) Neutrophils (NE), (B) Lymphocyte (LY), (C) Platelets (PLT) and (D) Plateletcrit (PCT) (n=4-6). *: $P<0.05, \#: P<0.001$, compared to untreated control. 

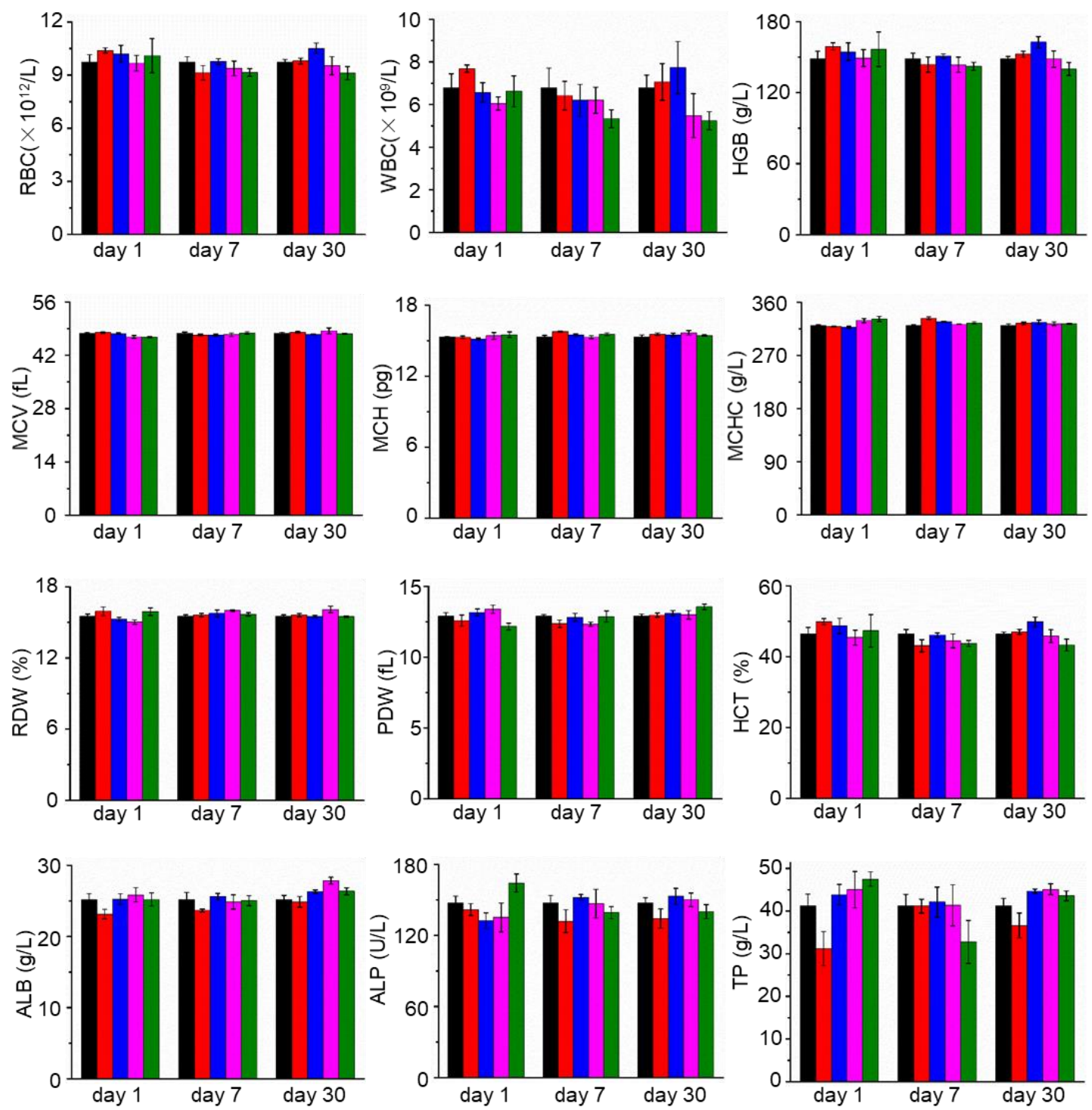

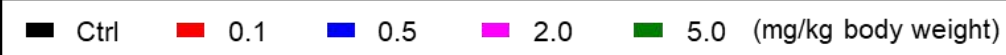

Figure S3. Hematological and biological assays for GO-exposed mice at various concentrations for different time. Red blood cell (RBC) count, white blood cell (WBC) count, hemoglobin (HGB) count, mean corpuscular volume (MCV), mean corpuscular hemoglobin $(\mathrm{MCH})$, mean corpuscular hemoglobin concentration (MCHC), red blood cell distribution width (RDW), platelet distribution width (PDW), hematocrit (HCT), albumin (ALB), alkaline phosphatase (ALP) and total protein (TP) $(n=4-6)$. 


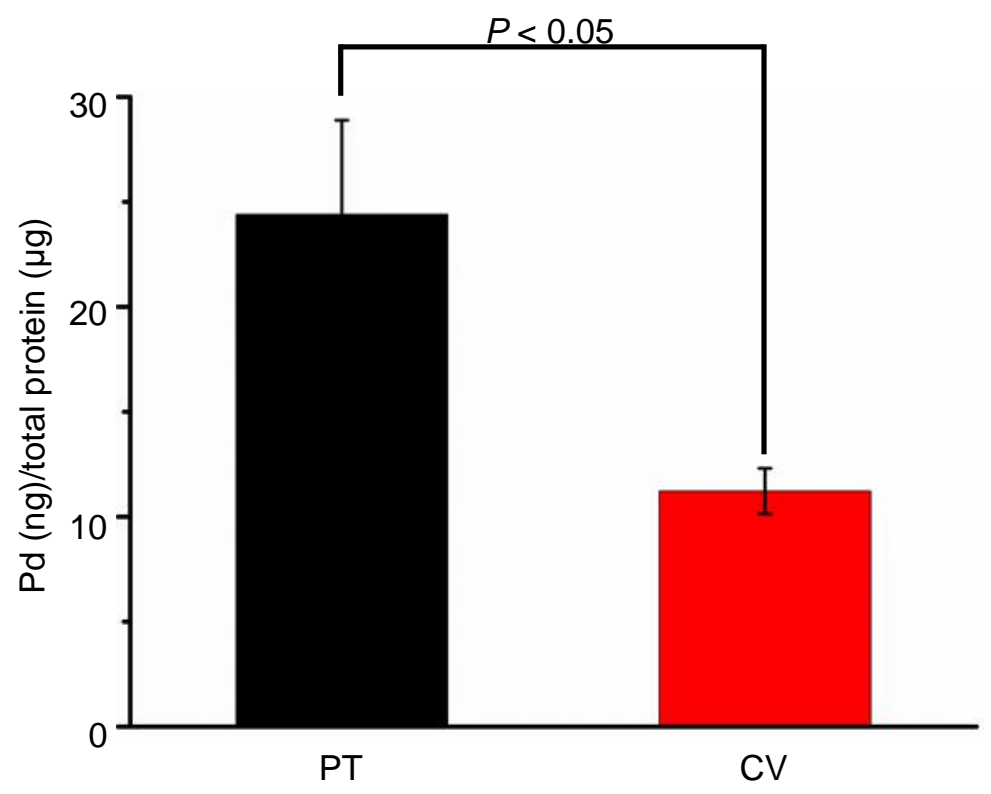

Figure S4. Pd nanosheets accumulated in the liver in a zonated manner. Quantification of Pd contents around the PT and CV areas $(\mathrm{n}=102)$. 
(A) Cyp1a1

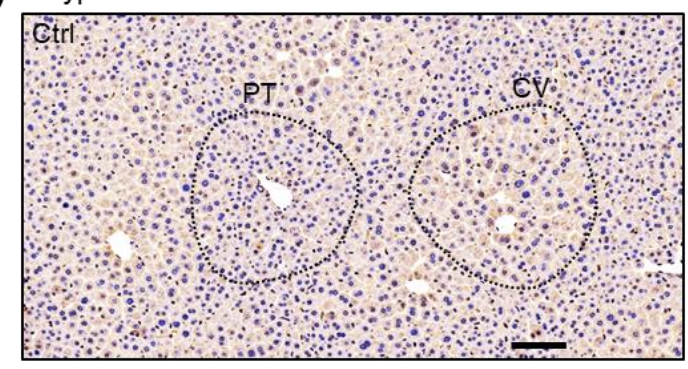

(B) Сурза7

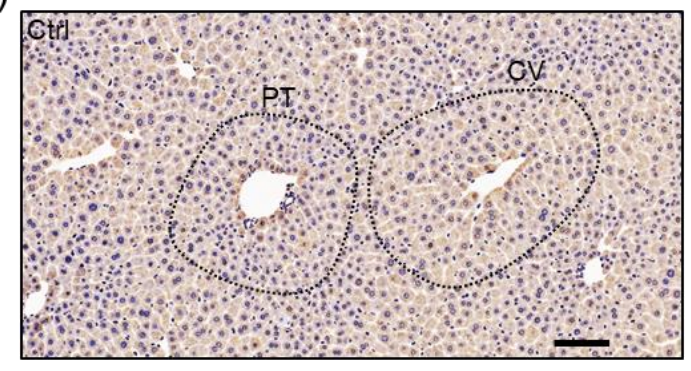

(C) Uba1

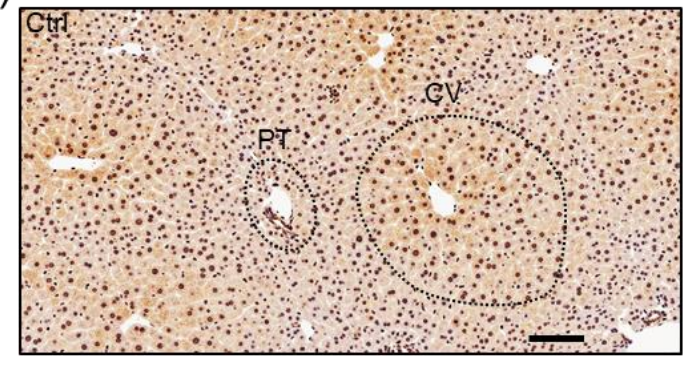

(D)

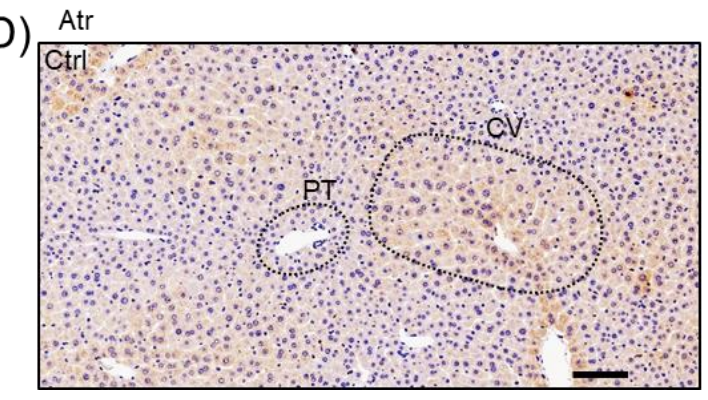

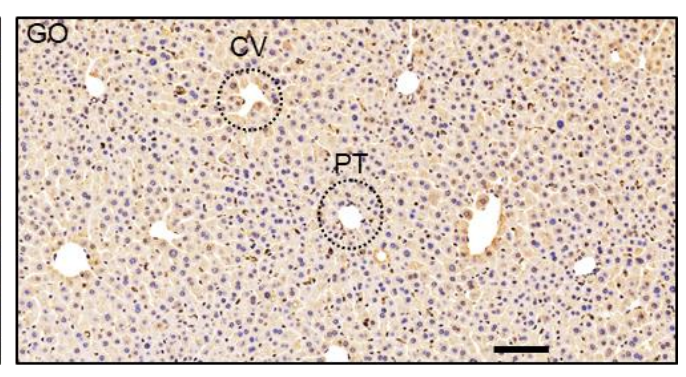
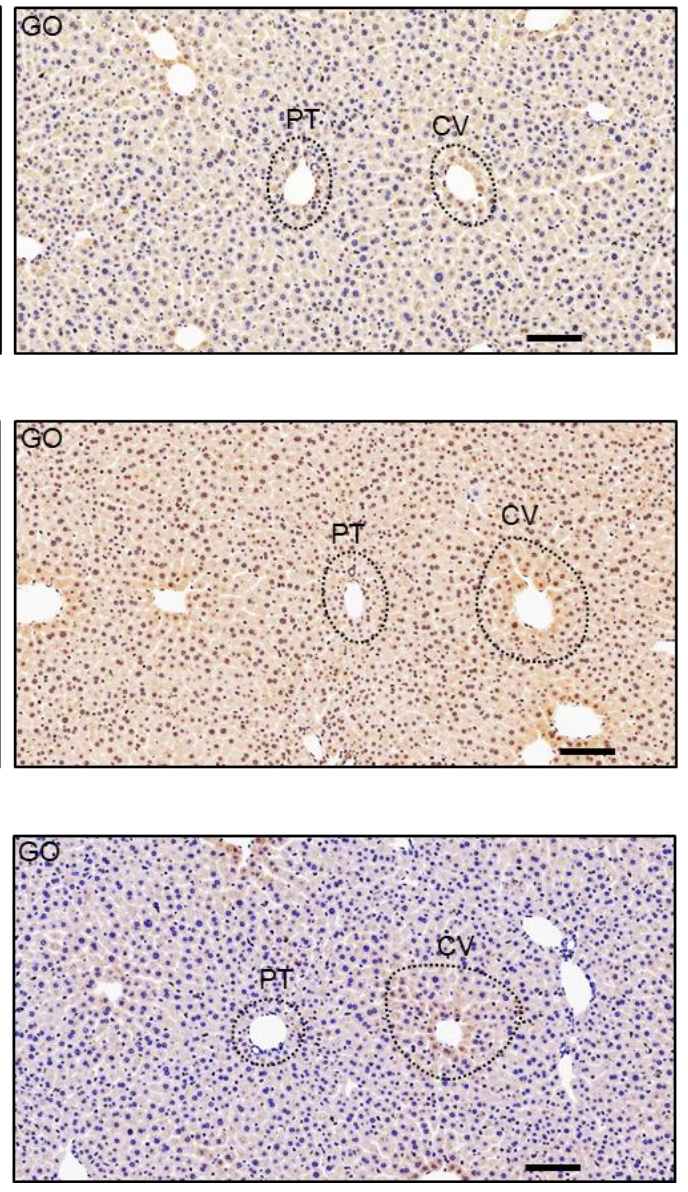

Figure S5. GO exposure caused zonated hepatotoxicity. Representative IHC examination of the liver sections from mice upon exposure to $2.0 \mathrm{mg} / \mathrm{kg}$ body weight $\mathrm{GO}$ materials for 7 days for (A) Cyp1a1, (B) Cyp3a7, (C) Uba1 and (D) Atr concentrations in PT and CV zones. Dotted circles denote PT and CV zones, and positive staining is shown in brown. Original magnification, $200 \times$. Scale bar, $100 \mu \mathrm{m}$. 
(A)

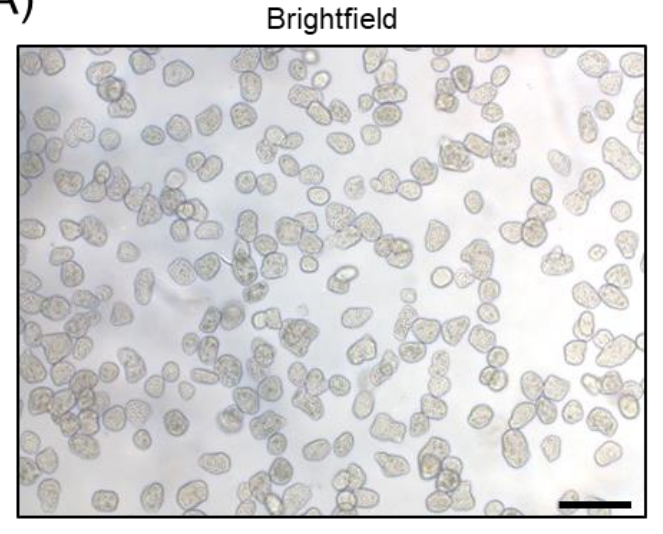

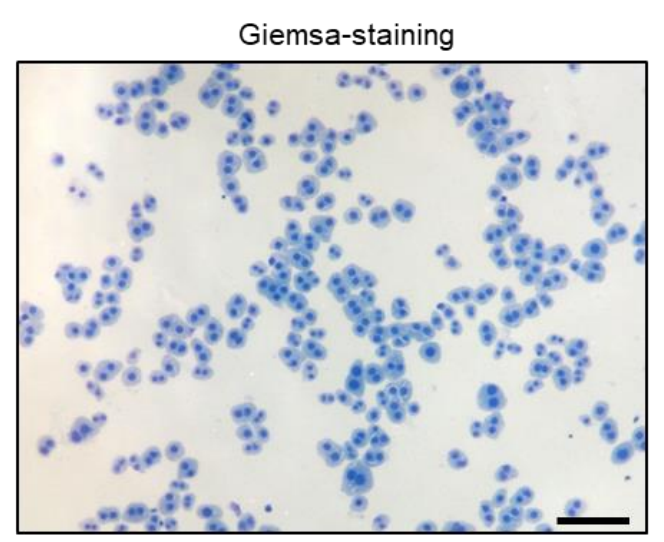

(B)
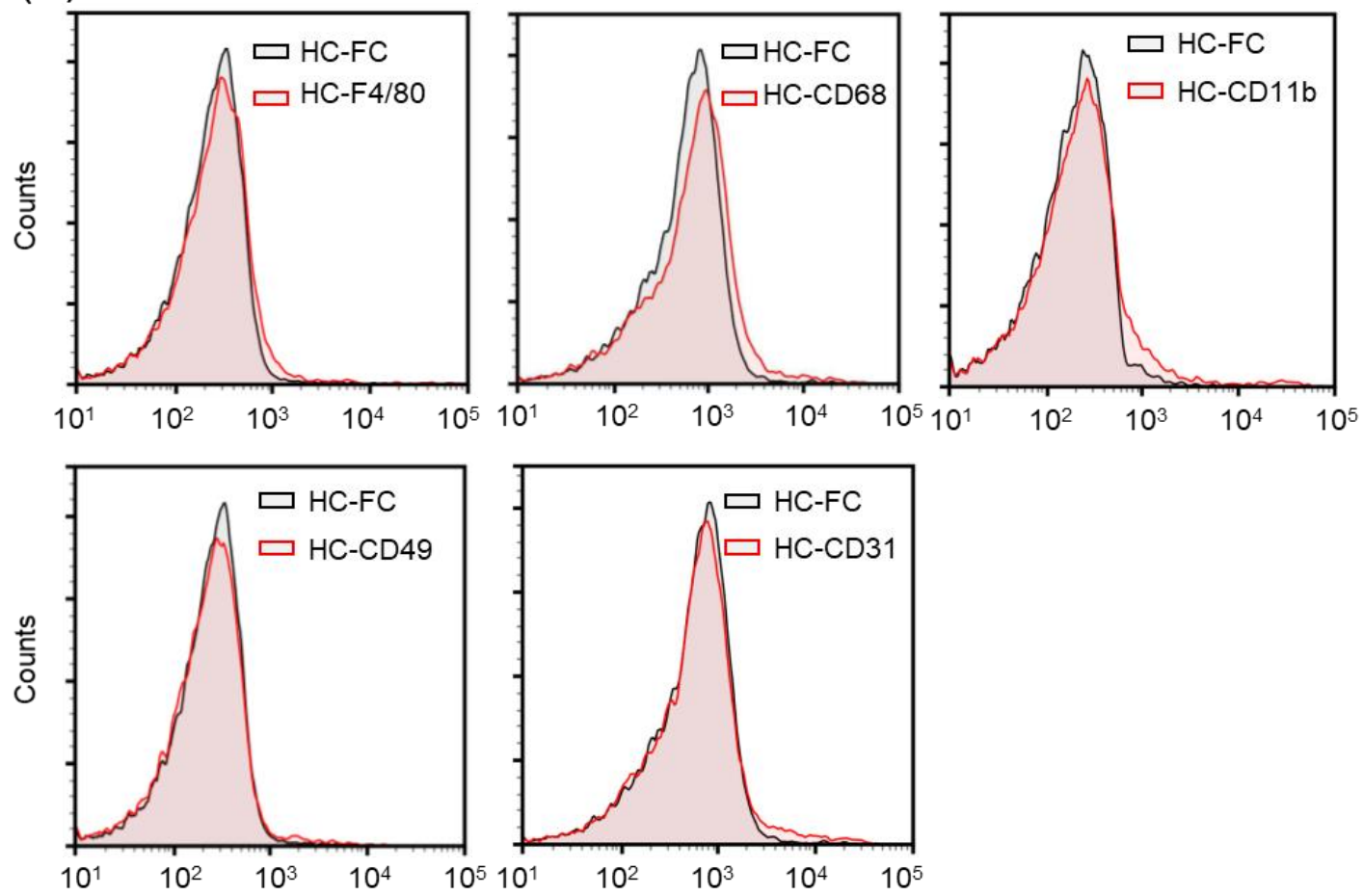

Figure S6. Examination of purified hepatocytes. Purified hepatocytes images were shown (A) in brightfield and Giemsa staining. Original magnification, 100×. Scale bar, $100 \mu \mathrm{m}$. (B) Flow cytometry analysis demonstrated no contamination of Kupffer cells and sinusoidal endothelial cells in purified hepatocytes using Abs against F4/80, CD11b, CD68 (3 markers for Kupffer cells), CD49 (a marker for sinusoidal endothelial cells) and CD31 (a marker for endothelial cells). 


\section{KEGG analysis of the differentially expressed genes}

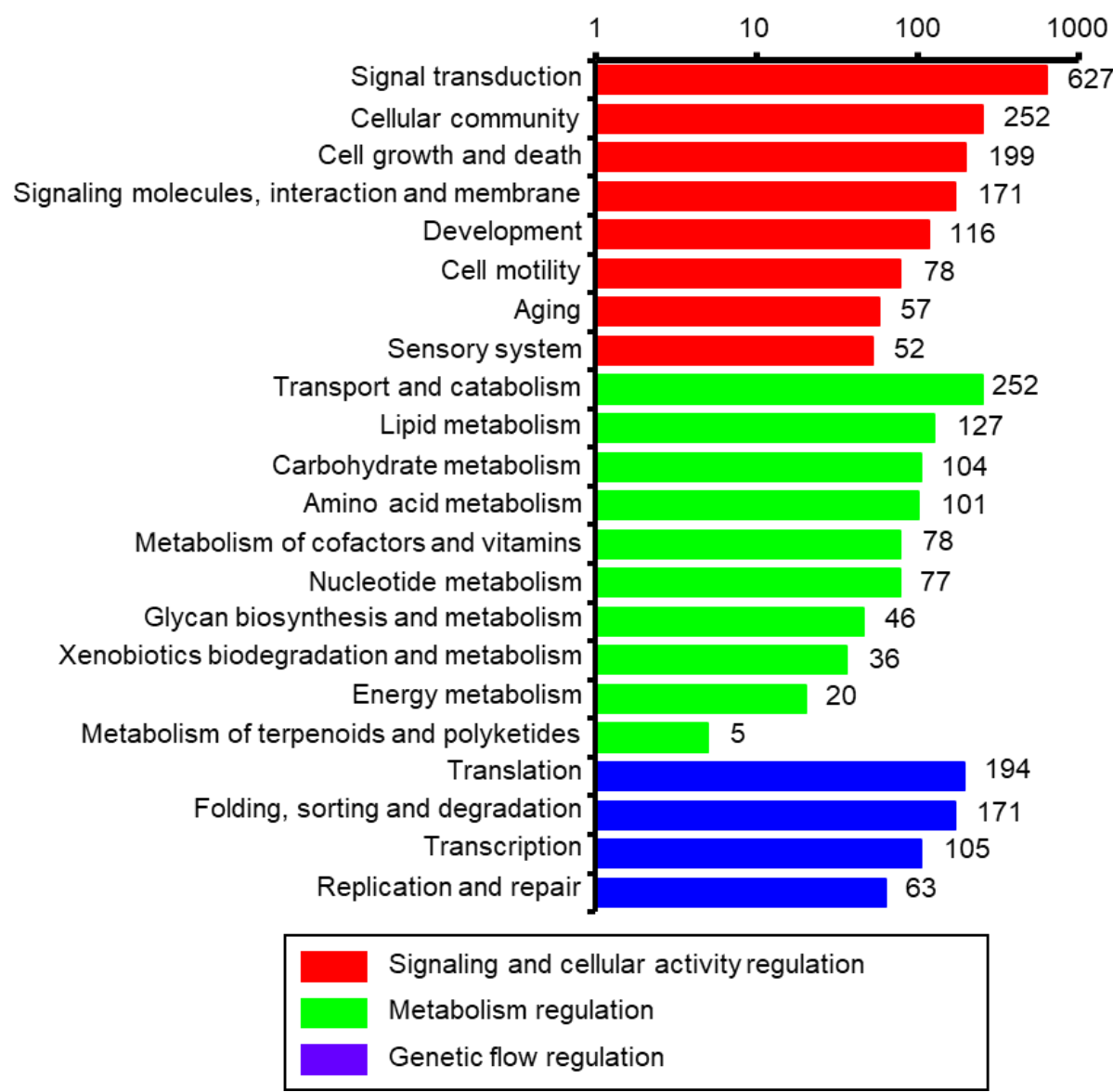

Figure S7. KEGG pathway analysis of the 4398 differentially expressed genes. 


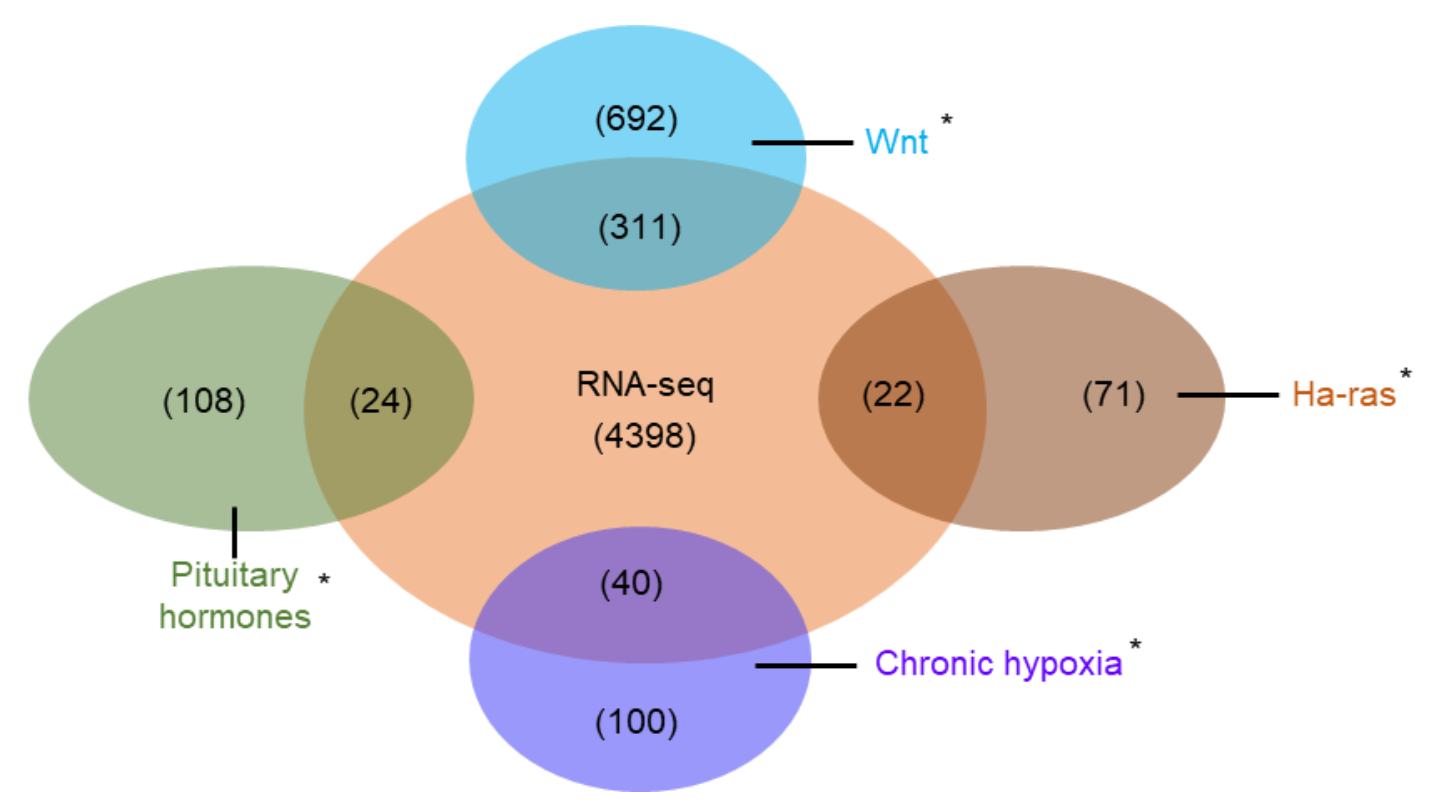

Figure S8. Differentially expressed genes from RNA-seq data were overlapped with Wnt-, Ha-ras-, chronic hypoxia- and pituitary hormone-target genes. *: genes from a previous study. ${ }^{52,53,73-75}$ 


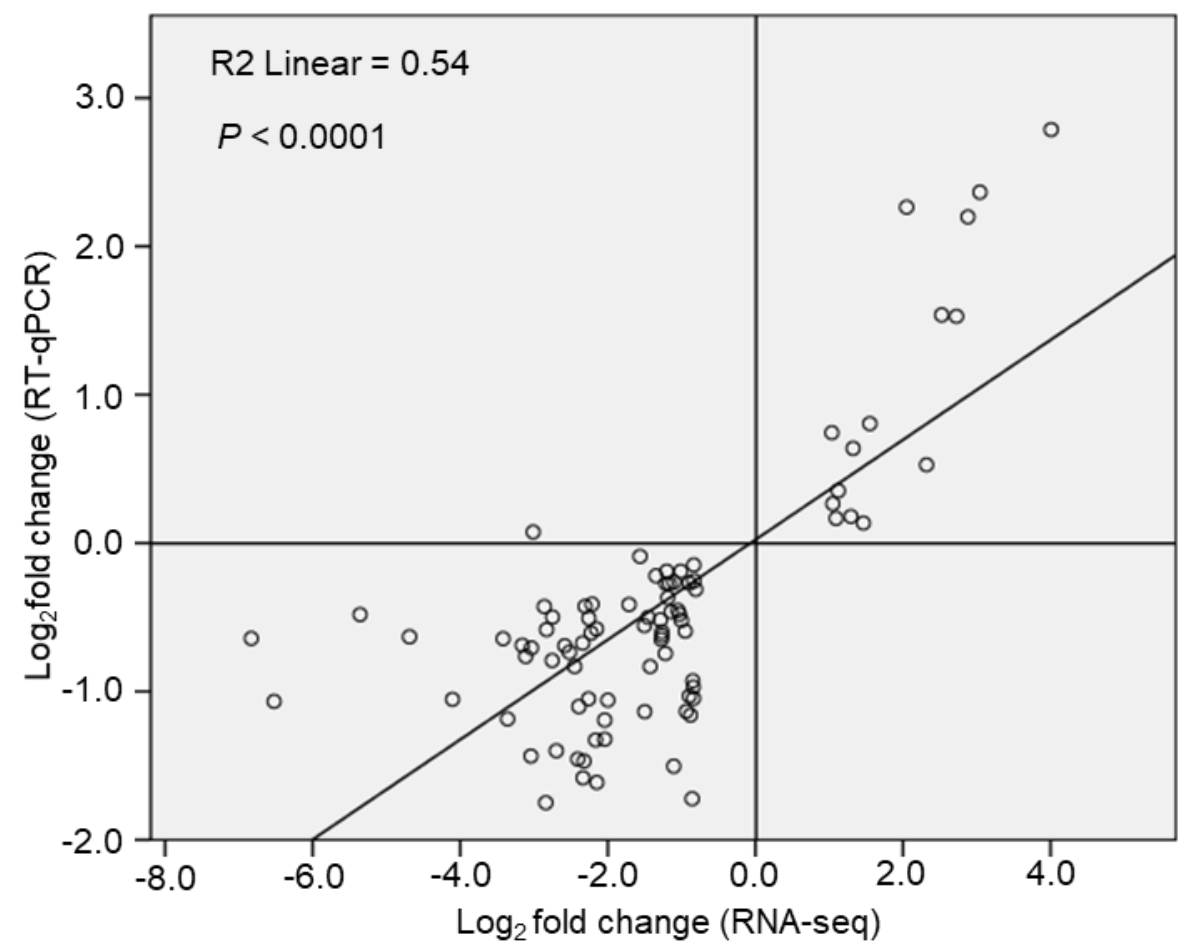

Figure S9. The correlation analysis between RNA-seq data and RT-qPCR results based on 87 differentially expressed genes. 


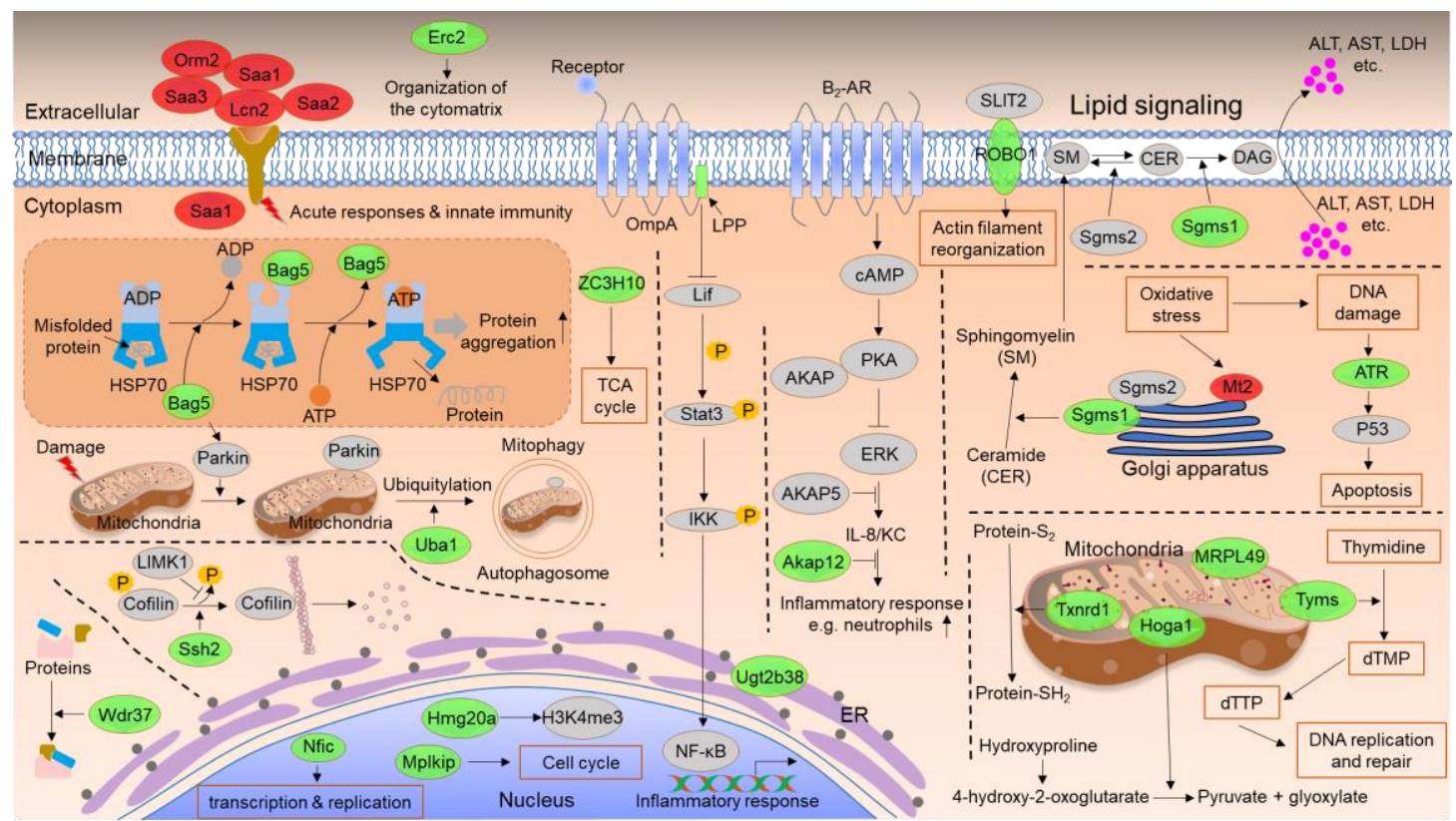

Figure S10. Overall diagram delineates the network of representative differentially expressed genes affected by GO exposure in hepatocytes. 


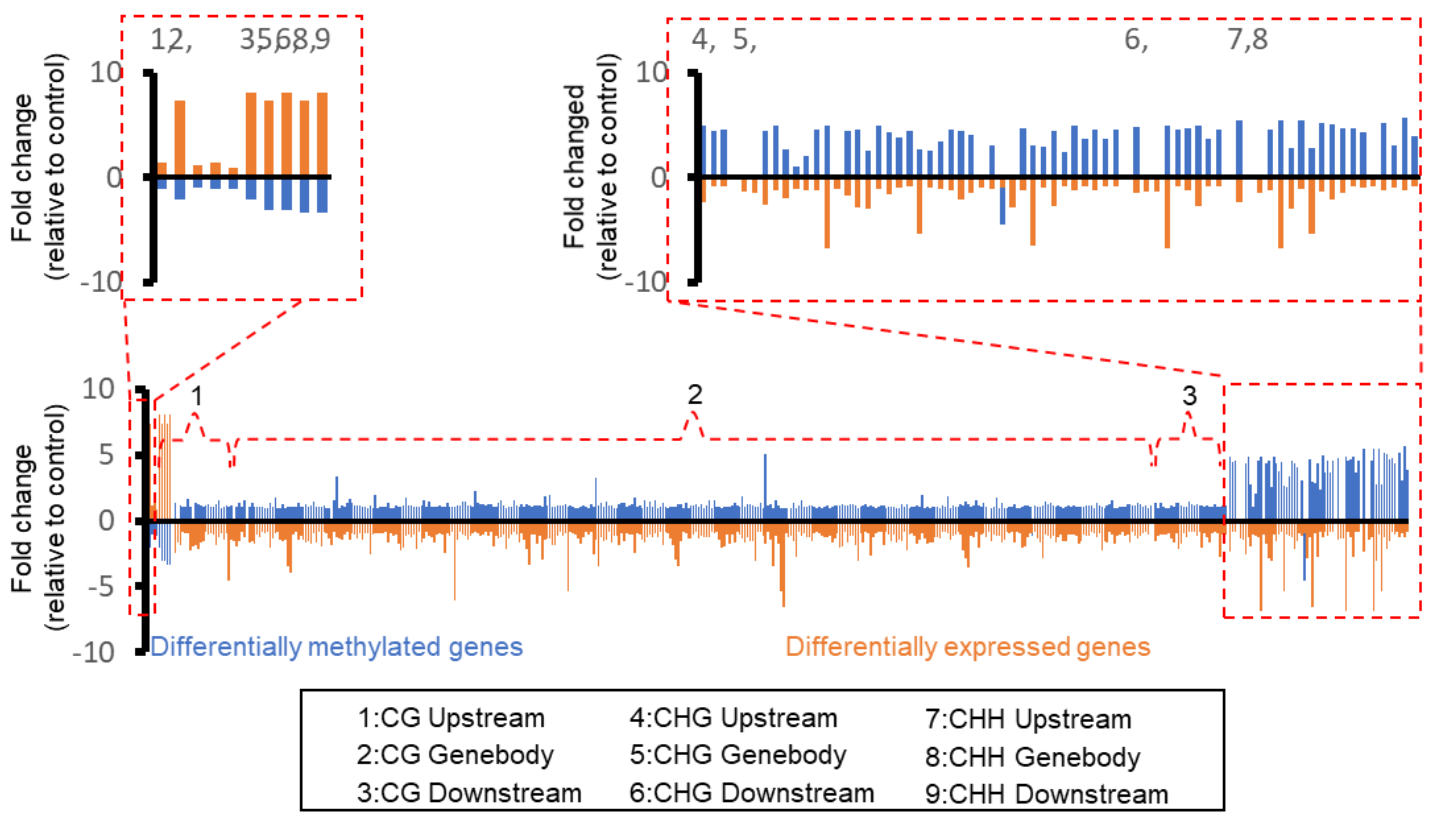

Figure S11. Correlation between the differentially methylated genes and the differentially expressed genes. 


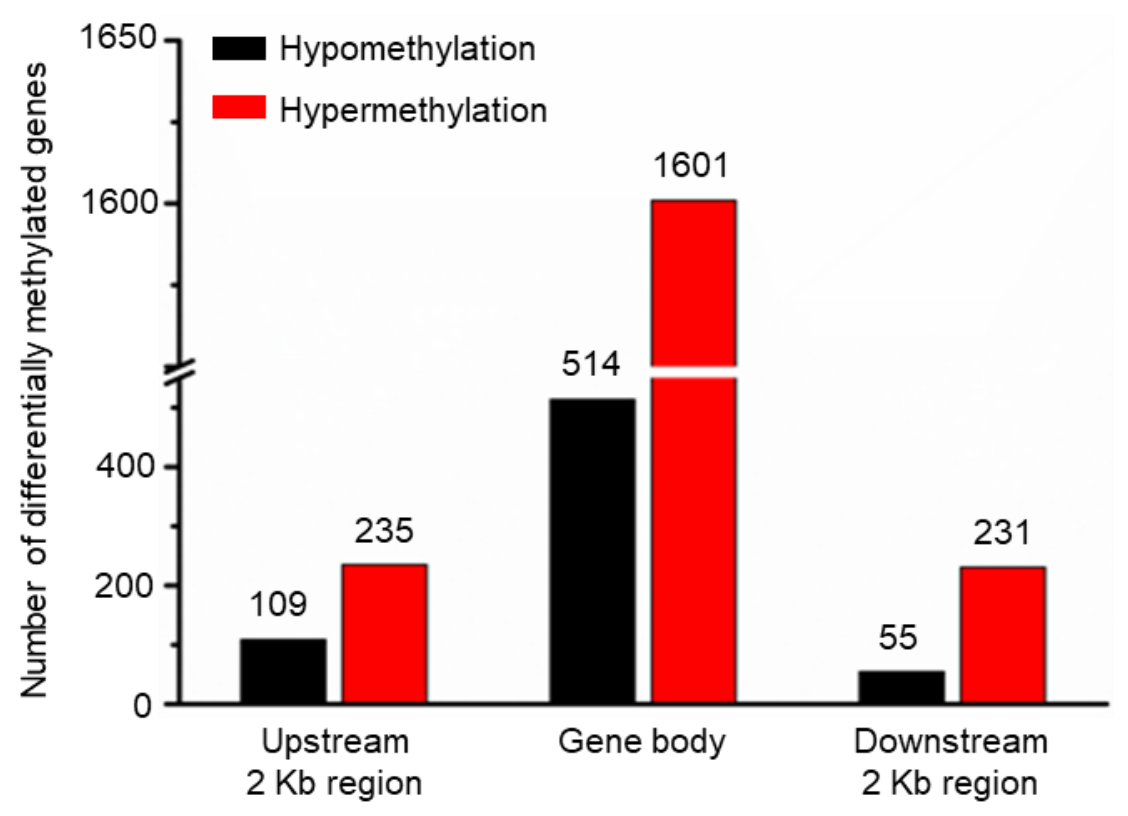

Figure S12. Distribution of the significantly differentially methylated genes. Significantly differentially methylated genes were divided into hypomethylation and hypermethylation with location in 3 regions as upstream $2 \mathrm{~Kb}$ region, gene body and downstream $2 \mathrm{~Kb}$ region, separated by transcription stating site (TSS) and transcription ending site (TES), respectively. 

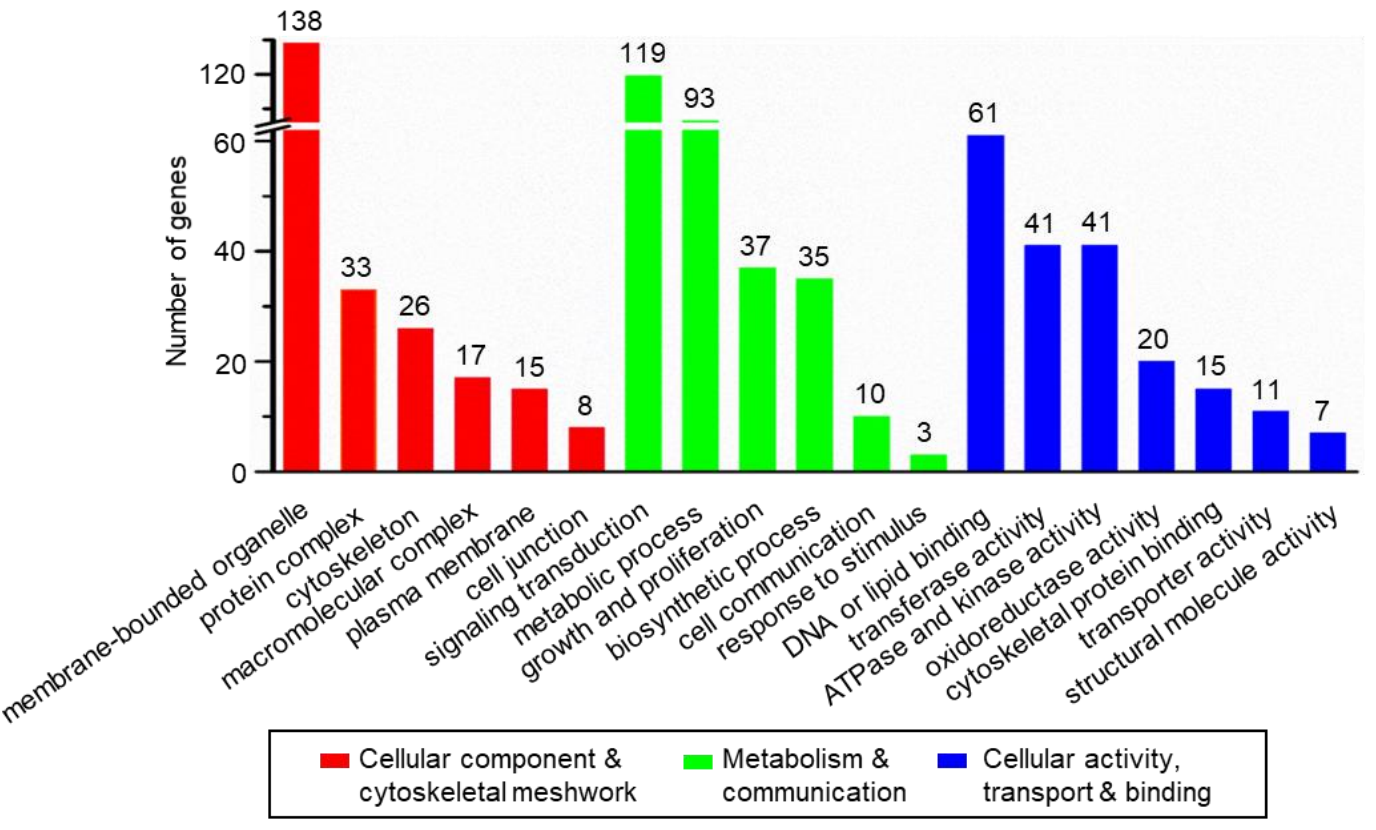

Figure S13. KEGG pathway analysis of 546 common genes between differentially expressed genes and differentially methylated genes. 
(A) TET1

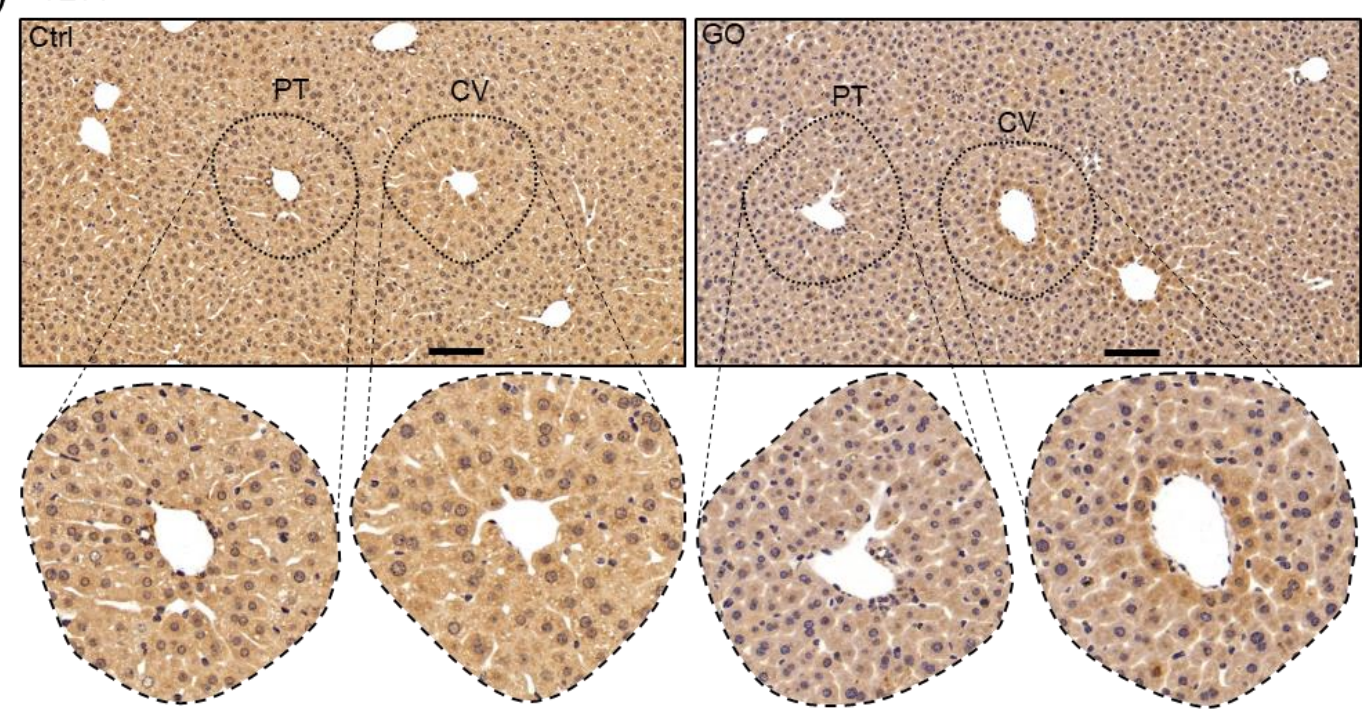

(B)

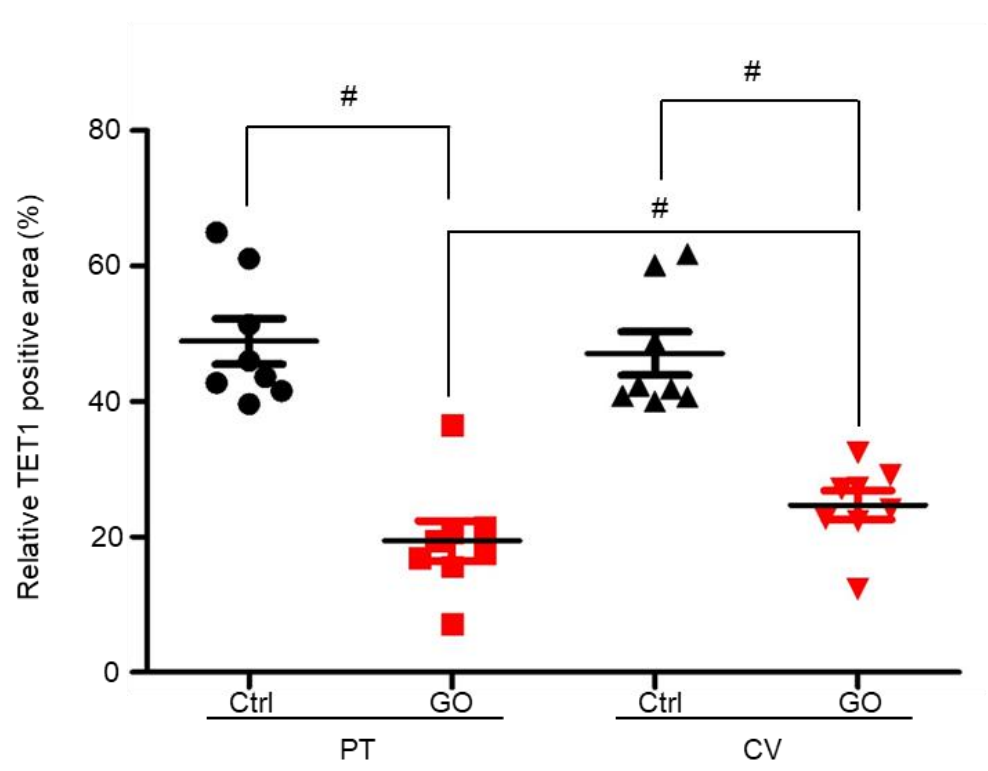

Figure S14. IHC analysis of TET1. (A) IHC examination of TET1 in liver sections from mice exposed to $\mathrm{GO}$ at $2.0 \mathrm{mg} / \mathrm{kg}$ body weight for 7 days. Original magnification, $200 \times$. Scale bar: 100 $\mu \mathrm{m}$. The lower panel shows the enlarged images of representative PT and CV zones. (B) Quantitative data of TET1 positive sites around PT and CV zones (n=8). \#: $P<0.001$. 


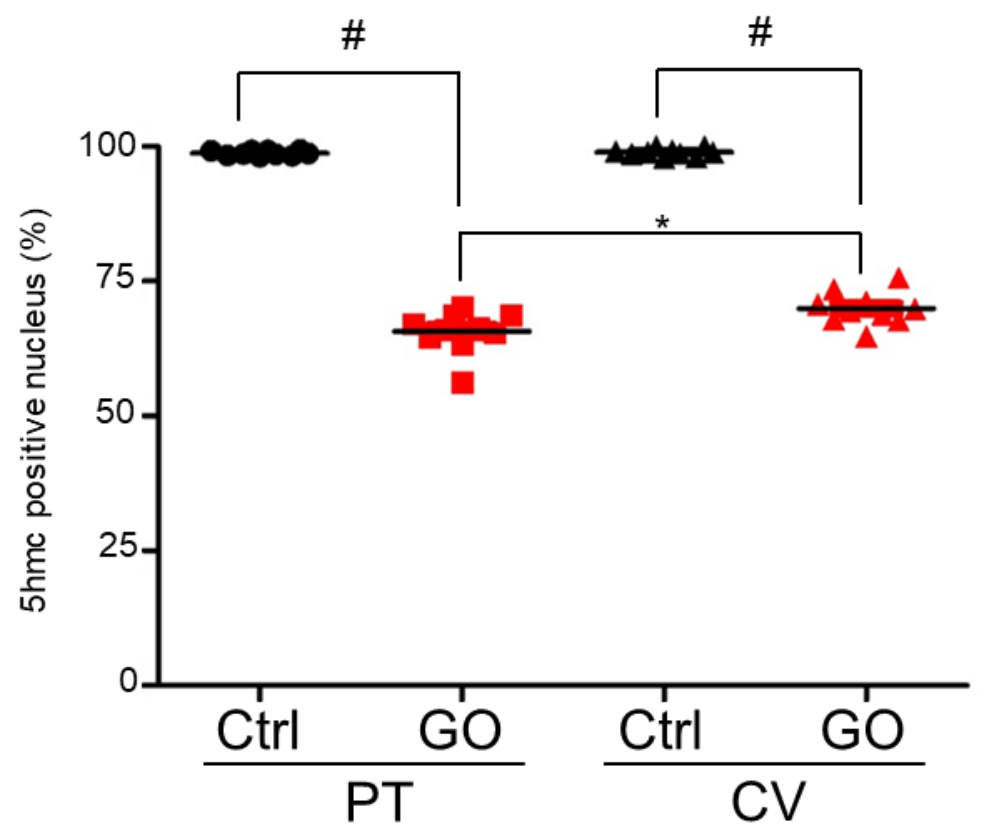

Figure S15. Quantitative analysis of $5 \mathrm{hmC}$ positive nuclei in liver sections with the IHC analysis of $5 \mathbf{h m C}(\mathrm{n}=10),{ }^{*}: P<0.05, \#: P<0.001$. 
Table S1. Quantitative data of functional groups based on the XPS data.

\begin{tabular}{c|c|c|c|c}
\hline Composition & $\mathrm{C}-\mathrm{C}$ & $\begin{array}{l}\mathrm{C}-\mathrm{OH} / \\
\mathrm{C}-\mathrm{O}-\mathrm{C}\end{array}$ & $\mathrm{C}=\mathrm{O}$ & $\mathrm{O}=\mathrm{C}-\mathrm{OH}$ \\
\hline $\mathrm{GO}$ & $0.46 \pm 0.01$ & $0.51 \pm 0.01$ & $0.02 \pm 0.00$ & $0.01 \pm 0.00$ \\
\hline
\end{tabular}


Table S2. The $\zeta$-potential $(\mathrm{mV})$ and hydrodynamic diameter $(\mathrm{nm})$ analysis of GO materials in water and culture medium (DMEM+10\% FBS).

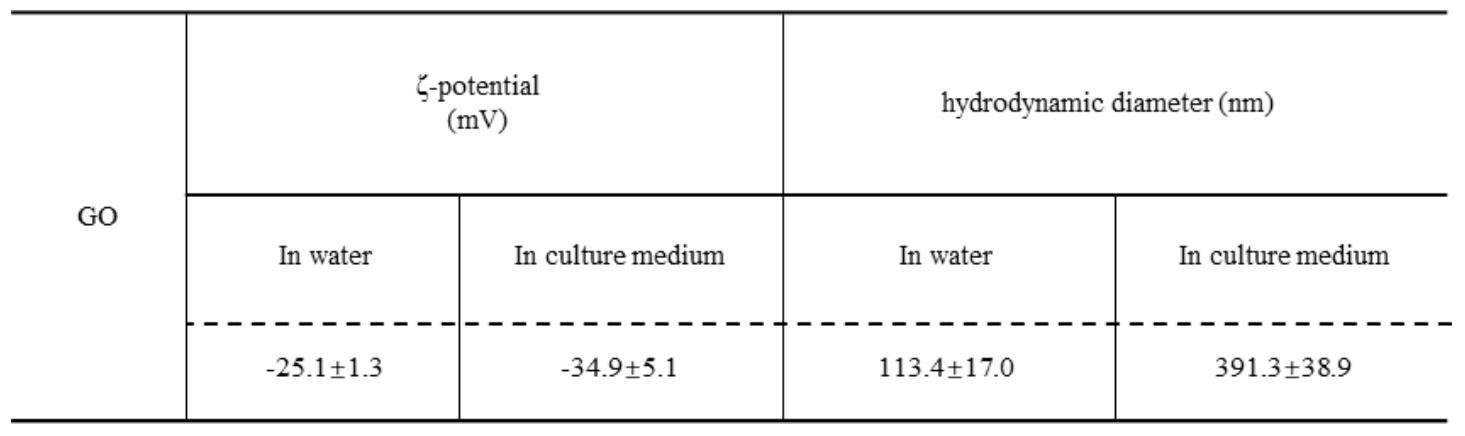


Table S3. Oligonucleotide primers for RT-qPCR analysis.

\begin{tabular}{|c|c|}
\hline Name & Sequence $\left(5^{\prime} \rightarrow 3^{\prime}\right)$ \\
\hline Nrep-F & GTCTTTCTAGCATGATGCCCTT \\
\hline Nrep-R & TATGCAAACGGTTCTTGGCTG \\
\hline Tyms-F & GGAAGGGTGTITGGAGGAGT \\
\hline Tyms-R & GCTGTCCAGAAAATCTCGGGA \\
\hline Park2-F & TCTTCCAGTGTAACCACCGTC \\
\hline Park2-R & GGCAGGGAGTAGCCAAGTT \\
\hline Ugt2b38-F & TGCGCCACAAAAGGGCTAA \\
\hline Ugt2b38-R & ACACAAGAGAGTAGGAAGCCG \\
\hline Nanog-F & TCTTCCTGGTCCCCACAGTाT \\
\hline Nanog-R & GCAAGAATAGTTCTCGGGATGAA \\
\hline Rhox8-F & CCAGGTCATGGGTCAATGCTT \\
\hline Rhox8-R & AGAAGAGACGACTGGGTGACT \\
\hline Ppp1r10-F & AGCTGGACACCCCATCTTTG \\
\hline Ppp1r10-R & GGACAGGGGCTGAATTGAGA \\
\hline Onecut1-F & GGCAACGTGAGCGGTAGTाT \\
\hline Onecut1-R & TTGCTGGGAGTTGTGAATGCT \\
\hline Smarca2-F & CTCCTGGACCAATTCTGGGG \\
\hline Smarca2-R & CATCGTTGACAGAGGATGTGAG \\
\hline Cplx2-F & AAGAGCGCAAGGCGAAACA \\
\hline Cplx2-R & TGGCAGATATTGAGCACTGTG \\
\hline Zfp488-F & CCATGACTTCCCAACTTAGGTCT \\
\hline Zfp488-R & CCСAATCTCATAACGGCCCA \\
\hline Ap1s3-F & GATTCTGAGCGCTCCATGCT \\
\hline Ap1s3-R & TGTGAAACACCTAGAGCCCC \\
\hline Olfr856-ps1-F & TGGCCATATGTCACCCCTTG \\
\hline Olfr856-ps1-R & ATAGGCCAGGCAGATTAGGC \\
\hline Wdr37-F & CCAGCTTTAAGACTACCACATCC \\
\hline Wdr37-R & ACAATAGGCTGTGTCCTTGTAAC \\
\hline Adh6-ps1-F & ACCGAGCACTTTGCCTTCT \\
\hline Adh6-ps1-R & TTGCAGGTAATTCTGCCGGT \\
\hline Mcmdc2-F & AGGCAGCCCTTGTCTATCTTG \\
\hline Mcmdc2-R & ACGTCACAGGGGTCTATTAGAAT \\
\hline Prnp-F & ATGGCGAACCTTGGCTACTG \\
\hline Prnp-R & CCTGAGGTGGGTAACGGTTG \\
\hline Gm10845-F & GTAGGTGCAGCTGGGTTACA \\
\hline Gm10845-R & GATGCCGTGTTGGGATGAGA \\
\hline Plac9a-F & GTGCAAAGGCGGTTAGACATT \\
\hline Plac9a-R & GCCCTGTGGGAAGGTTTGA \\
\hline Obp2a-F & AGAGCCTGGCAAATACACCG \\
\hline Obp2a-R & AATGTAGTGCTCATTCACCGAC \\
\hline$D d \times 46-F$ & ATGTCTGGACGAGATTTGATGG \\
\hline Ddx46-R & AGCTATTGGTCCCTCTCCTTC \\
\hline
\end{tabular}




\begin{tabular}{|c|c|}
\hline Name & Sequence $\left(5^{\prime} \rightarrow 3^{\prime}\right)$ \\
\hline Lce3a-F & GTAGTGCCATGTTGGCTTGTT \\
\hline Lce3a-R & AAGCAACCAGGCTTTTGGGAA \\
\hline Arglu1-F & TCCCGCTCTAAGCACACCA \\
\hline Arglu1-R & CCGGTTCCGTTIGCTCTCC \\
\hline Mplkip-F & CAGTAATCACTTAGACTITGAGGGT \\
\hline Mplkip-R & TाTGGGCAGAGCACTGAACT \\
\hline Mrgpra6-F & CCACATCATAGCTTCCACAGAG \\
\hline Mrgpra6-R & TGCAGAGCAATAGAGACAGGA \\
\hline Gm9159-F & GAGTGGTTGCCAGCTACAGA \\
\hline Gm9159-R & CCCTAAGAGCTTGCACCCTT \\
\hline D5Ertd615e-F & TAGGATGACAAGCCACCTGC \\
\hline D5Ertd615e-R & ACAGGAACTCCATCGCAGAC \\
\hline Orm1-F & CGAGTACAGGCAGGCAATTCA \\
\hline Orm1-R & ACCTATTGTTGAGACTCCCGA \\
\hline Lcn2-F & TGGCCCTGAGTGTCATGTG \\
\hline Lcn2-R & СTCTTGTAGCTCATAGATGGTGC \\
\hline Saa2-F & TAGACCACCAGATCTGCCCA \\
\hline Saa2-R & TCATGTCAGTGTAGGCTCGC \\
\hline Saa1-F & ATCACCAGATCTGCCCAGGA \\
\hline Saa1-R & CCTTGGAAAGCCTCGTGAACA \\
\hline Orm2-F & GTGTCCTAAGCATGGCACTG \\
\hline Orm2-R & GCCGGTAATCAGGGTTTAGG \\
\hline Saa3-F & TGCCATCATTCTTGCATCTTGA \\
\hline Saa3-R & CCGTGAACTTCTGAACAGCCT \\
\hline Cyp2c39-F & GAGGAAGCATTCCAATGGTAGAA \\
\hline Cyp2c39-R & TGTGAAGCGCCTAATCTCTTTC \\
\hline Mt2-F & GCCTGCAAATGCAAACAATGC \\
\hline Mt2-R & AGCTGCACTTGTCGGAAGC \\
\hline Mt1-F & AAGAGTGAGTTGGGACACCTT \\
\hline Mt1-R & CGAGACAATACAATGGCCTCC \\
\hline Gdf15-F & CTGGCAATGCCTGAACAACG \\
\hline Gdf15-R & GGTCGGGACTTGGTTCTGAG \\
\hline Apcs-F & AGACAGACCTCAAGAGGAAAGT \\
\hline Apcs-R & AGGTTCGGAAACACAGTGTAAAA \\
\hline Saa4-F & CCAGGGCCTCCTAAACCGATA \\
\hline Saa4-R & CTCGGGTCGGAAGTGATTGG \\
\hline Efna1- $F$ & СTTCACGCCTTTATCTTGGGC \\
\hline Efna1-R & TGGGGATTATGAGTGATTITGCC \\
\hline Itih3-F & TGCTCACAATGTTGTCACCAC \\
\hline Itih3-R & TGTCTTGGGCAGTTCCACATC \\
\hline Insig2-F & GGAGTCACCTCGGCCTAAAAA \\
\hline Insig2-R & CAAGTTCAACACTAATGCCAGGA \\
\hline Asrgl1-F & CGGAGCAAGCAACATCTCAG \\
\hline Asrgl1-R & CTGGGTCGTITCCAGGACA \\
\hline
\end{tabular}




\begin{tabular}{|c|c|}
\hline Name & Sequence $\left(5^{\prime} \rightarrow 3^{\prime}\right)$ \\
\hline Erc2-F & ATGTACGGGAGCGCAAGAAC \\
\hline Erc2-R & GGCTGCATTAAGGGACTGGATA \\
\hline Gtpbp3-F & ССTACATTGACTTCGGAGAGGA \\
\hline Gtpbp3-R & CACGCCTAGCATCTCGCAG \\
\hline Kif1b-F & GTCAATCGAATGAACGACCTGG \\
\hline Kif1b-R & GCCGATGCAAAAAGTTGAACTG \\
\hline Pabpn1-F & GGAGCTAGAAGCGATCAAAGC \\
\hline Pabpn1-R & ATTGCCAACGTAGATAGAGCG \\
\hline Robo1-F & AACGGGAGAGTGAAGTCGC \\
\hline Robo1-R & TCTTTCCTCCATCGAACTGTAGG \\
\hline Gemin4-F & GGAGATGACTATTCTGCATGGG \\
\hline Gemin4-R & GCCACCGAGTATCAGTGTCAG \\
\hline Mrpl49-F & GATAACCCCGGCTTGTGGAG \\
\hline Mrpl49-R & GGCTGCCAACCACTAGGAG \\
\hline Zc3h10-F & GGGACAGCTATGCCAACGG \\
\hline Zc3h10-R & GCCTCGTTTGCAGACGTTC \\
\hline $\mathrm{Ar}-\mathrm{F}$ & CTGGGAAGGGTCTACCCAC \\
\hline Ar-R & GGTGCTATGTTAGCGGCCTC \\
\hline Atr-F & ACTIITACGGATTGCAGCAACT \\
\hline Atr-R & ССАТТССАТААССТСАСССАС \\
\hline Cep170-F & TGCCTGCTAGTTCTTCATTCAAG \\
\hline Cep170-R & CCGTGCCTGAGGAAGTCAC \\
\hline Cep63-F & ACCAAAAGTTITCGAGAGGATCG \\
\hline Cep63-R & GATTATCTCCGACTGTTCAGCC \\
\hline Dbil5-F & CCCAGGGCGACTGTAACATC \\
\hline Dbil5-R & GCAATGTAGATCCTCATGGCAT \\
\hline Dmd-F & CGAGACCCAAACCACTTGTTG \\
\hline Dmd-R & GGTCAGCTAAAGACTGGTAGAGC \\
\hline Eid3-F & TTGAGAGAATCTTGGGATTGCTG \\
\hline Eid3-R & CACAGTGCGAGAGAATGAGTTा \\
\hline Fam46c-F & GCGTGCTCAACTGGGATCA \\
\hline Fam46c-R & CAACCCAAGCCGTTGTCTT \\
\hline Glod4-F & GGGAAGTGGAGTAAAACAATGGT \\
\hline Glod4-R & CTTGGCTAGAAGCAAGCGTGA \\
\hline Hk1-F & CGGAATGGGGAGCCTTTGG \\
\hline Hk1-R & GCCTTCCTTATCCGTTTCAATGG \\
\hline Hmg20a-F & CGCCCCTGTTGCAGATGA \\
\hline Hmg20a-R & CTCTGGGTTGGTTGTTGATGT \\
\hline Hoga1-F & GCAGGGGCTTGTCTAGGAATG \\
\hline Hoga1-R & CAGGAACGGAAACTCTCCAGT \\
\hline Hspa2-F & GCGTGGGGGTATTCCAACAT \\
\hline Hspa2-R & TGAGACGCTCGGTGTCAGT \\
\hline Kpna4-F & CCAGTGATCGAAATCCACCAA \\
\hline Kpna4-R & CGTTGTTCAGACGTTCCAGAT \\
\hline
\end{tabular}




\begin{tabular}{|c|c|}
\hline Name & Sequence $\left(5^{\prime} \rightarrow 3^{\prime}\right)$ \\
\hline Lpp-F & CAGCATCTTGGCTGATTTGGA \\
\hline Lpp-R & GTTGCGGACTTCTGGTTGC \\
\hline Mapk6-F & TCGAAGACTTGACCACGATAACA \\
\hline Mapk6-R & GCAGTACGTTTGCAGAATGGATA \\
\hline Nol8-F & CCAGCAATCTTGATAGATGCCC \\
\hline Nol8-R & CCGAACTGATTITGTAGGTCTGT \\
\hline Odf2-F & CGCGGTCGGTTGTCTCAAA \\
\hline Odf2-R & AGCACCGTGTTCTCATGCTC \\
\hline $\mathrm{Pcm1-F}$ & CCACAGGAGGAGGTCCTTITG \\
\hline Pcm1-R & CATATTGTTGAGTCGGTCATCCA \\
\hline Sgms1-F & TTGGCACGCTGTACCTGTATC \\
\hline Sgms1-R & CAGTCTCCAAAGAGCTTCGGA \\
\hline Sord-F & GCTAAGGGCGAGAACCTGTC \\
\hline Sord-R & CATGCTCCCAGTAGTGAACATC \\
\hline Spa17-F & AGGGCTGACACGGGAGATT \\
\hline Spa17-R & CCСATTCTGCTGGATCAAAGC \\
\hline Ssh2-F & TGGTGGGTTCAGCGTATCAAC \\
\hline Ssh2-R & CCGATGAGACTGCACATCCTG \\
\hline Tdrkh-F & TCCACTGAACGAACTTCATGG \\
\hline Tdrkh-R & TCTCTGCTTTCCCTATATCGACG \\
\hline Trim27-F & GGAGCAAATCCAGAACCGACT \\
\hline Trim27-R & GCCCCGTTGATGCTGTTATAG \\
\hline Trrap-F & AGCTCTCACGGATGTGAATACA \\
\hline Trrap-R & CCTGTGGAGTATCTCAAGTACCA \\
\hline Tubb4b-F & CACTTACCACGGAGATAGCGA \\
\hline Tubb4b-R & ACCTTCTGTGTAGTGCCCCTT \\
\hline Txnrd1-F & CCCACTTGCCCCAACTGTT \\
\hline Txnrd1-R & GGGAGTGTCTTGGAGGGAC \\
\hline Uba1-F & TGACAGAATCCTACAGCTCTAGC \\
\hline Uba1-R & CACTGAAGAGTGTGTTCGATGG \\
\hline Ybx3-F & GCGGCAGCAGTGAAGGATT \\
\hline Ybx3-R & ACGGTCAAAGTTCTGTCCCAC \\
\hline Akap12-F & CTGTCTGCCGTCAATGGTGTA \\
\hline Akap12-R & TGAAGCAGGGATCTGTTCGAT \\
\hline Nfic-F & CCGGCATGAGAAGGACTCTAC \\
\hline Nfic-R & TTCTTCACCGGGGATGAGATG \\
\hline Bag5-F & ААССААСАССССТССАТТАGТ \\
\hline Bag5-R & GCCTCTTGTAGTTCTTGTCATCC \\
\hline Lyar-F & ATGTGGTGAATCCGTGAAGAAAA \\
\hline Lyar-R & TTGCCTCCGTACTTCTGACCT \\
\hline Pam-F & CTGGGGTCACACCTAAAGAGT \\
\hline Pam-R & ATGAGGGCATGTTGCATCCAA \\
\hline Rnf19a-F & TGCCTITACGGCATTCTAAAGA \\
\hline Rnf19a-R & AGGATTAAACCGTTCAGTGCAT \\
\hline
\end{tabular}

